\title{
Retromer in Synaptic Function and Pathology
}

\author{
Lennart Brodin ${ }^{1 *}$ and Oleg Shupliakov ${ }^{1,2}$ \\ ${ }^{1}$ Department of Neuroscience, Karolinska Institutet (KI), Stockholm, Sweden, ${ }^{2}$ Institute of Translational Biomedicine, \\ St. Petersburg University, St. Petersburg, Russia
}

The retromer complex mediates export of select transmembrane proteins from endosomes to the trans-Golgi network (TGN) or to the plasma membrane. Dysfunction of retromer has been linked with slowly progressing neurodegenerative disorders, including Alzheimer's and Parkinson's disease (AD and PD). As these disorders affect synapses it is of key importance to clarify the function of retromer-dependent protein trafficking pathways in pre- and postsynaptic compartments. Here we discuss recent insights into the roles of retromer in the trafficking of synaptic vesicle proteins, neurotransmitter receptors and other synaptic proteins. We also consider evidence that implies synapses as sites of early pathology in neurodegenerative disorders, pointing to a possible role of synaptic retromer dysfunction in the initiation of disease.

Keywords: retromer, VPS35, synaptic vesicle, endosome, ionotropic receptor, G protein-coupled receptor, Alzheimer's disease, Parkinson's disease

OPEN ACCESS

Edited by:

Francisco J. Barrantes, Laboratorio de Neurobiología

Molecular, Instituto de

Investigaciones Biomédicas (BIOMED UCA-CONICET), Argentina

Reviewed by: Eckart D. Gundelfinger, Leibniz Institute for Neurobiology (LG), Germany Oksana Sorokina, University of Edinburgh, United Kingdom

${ }^{*}$ Correspondence: Lennart Brodin lennart.brodin@ki.se

Received: 16 August 2018 Accepted: 03 October 2018 Published: 24 October 2018

Citation:

Brodin L and Shupliakov O (2018) Retromer in Synaptic Function and Pathology.

Front. Synaptic Neurosci. 10:37. doi: 10.3389/fnsyn.2018.00037
The retromer protein complex also referred to as retromer, is a critical component of the endosomal protein sorting machinery. This complex recognizes specific transmembrane proteins and exports them by forming tubules to promote transport. Of three endosomal export destinations (Figure 1A)-retrograde transport to the trans-Golgi network (TGN), recycling to the plasma membrane, and traffic to lysosomes-the former two are controlled by retromer (Seaman, 2012; McNally and Cullen, 2018). Retromer is composed of two main parts, the cargo-selection complex (CSC) and the tubulation module. The CSC consists of three largely globular proteins, VPS35, VPS26 and VPS29, named after the vacuolar protein sorting genes in yeast. The stability of the CSC depends on VPS35 and hence knockout/knockdown of this protein is commonly used to disrupt retromer function. The tubulation module comprises heterodimers of the BAR domain-containing sorting nexins SNX1/SNX2 and SNX5/SNX6 (Seaman, 2012; Mukadam and Seaman, 2015). Recent structural studies indicate that the CSC promotes tubule formation by directing the distribution of SNX proteins on the membrane surface (Kovtun et al., 2018).

Retromer function depends on a set of accessory proteins. Among the most well characterized are SNX3 and Rab7a that mediate recruitment of the CSC to the endosomal membrane, and the GTPase activating protein TBC1D5, which acts on Rab7a to inhibit the recruitment process (Seaman, 2012; Seaman et al., 2018). The CSC also interacts with the WASH complex that mediates actin polymerization, which serves to constrain retromer cargo and/or signaling proteins at discrete endosomal regions. It may also take part in the scission of tubules (Seaman and Freeman, 2014). The WASH complex is associated with SNX1 via the DNAJ protein RME8 (Seaman and Freeman, 2014). Another accessory protein is the dynamin-like ATPase EHD. It is thought to stabilize endosomal tubules and take part in their scission (Zhang et al., 2012). In addition to those proteins, SNX27 has been implicated specifically in the trafficking of retromer cargos to the plasma membrane (Temkin et al., 2011). Contrary to yeast in which the CSC and the tubulation module are tightly associated, the two modules in animal cells are more loosely associated and can even act independently of each other, creating a greater functional diversity (Seaman, 2012, 2017). 
A

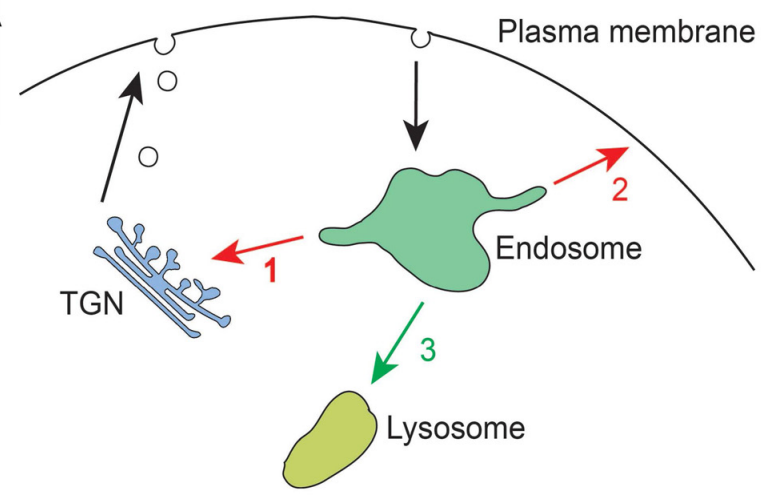

C
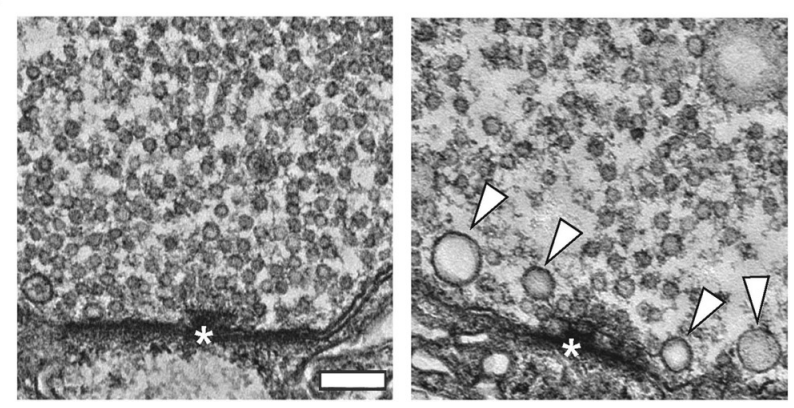

B

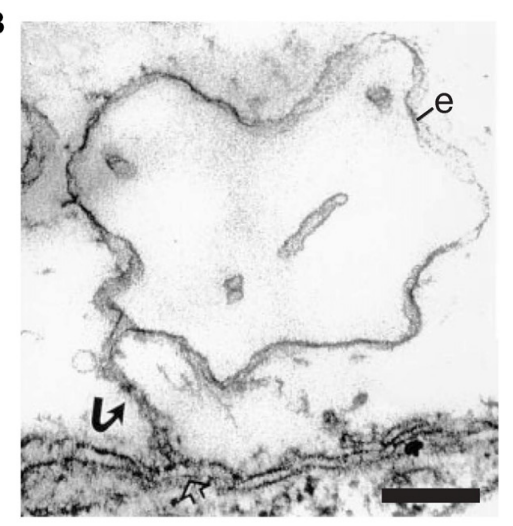

D

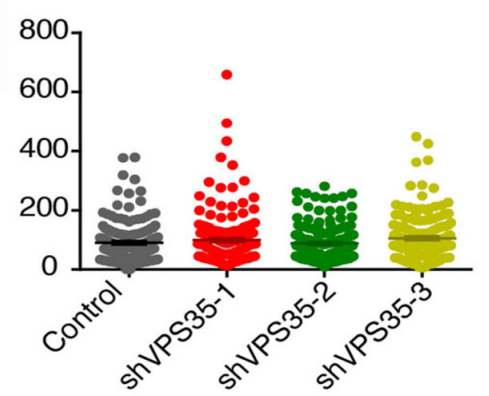

FIGURE 1 | Retromer and the trafficking of synaptic vesicle proteins. (A) Endosomal trafficking pathways in animal cells. Proteins entering endosomes can be exported retrogradely to the trans-Golgi network (TGN; 1), or recycled back to the plasma membrane (2), or transferred to lysosomes via late endosomes for degradation (3). Retromer controls pathways 1 and 2. (B) "False" endosome induced by synaptic activity at a presynaptic release site. The electron micrograph shows an endosome-like object (e) in a repetitively stimulated lamprey reticulospinal axon. Note the connection with the plasma membrane via a narrow stalk (arrow) revealing that the endosome-like object is in fact an invagination of the plasma membrane. Bar $=200 \mathrm{~nm}$ (reproduced from Gad et al., 1998 with permission). (C) Deletion of VPS35 in Drosophila alters synaptic vesicles in larval motor terminals. The left micrograph shows a control motor terminal. The right micrograph shows a terminal from a larva deficient in VPS35 and expressing a non-rescuing D620N mutant form of VPS35. Note the decreased number of synaptic vesicles and the occurrence of some large vesicles (arrowheads). Bar $=100 \mathrm{~nm}$ (reproduced from Inoshita et al., 2017 with permission). (D) Knockdown of retromer in mouse hippocampal neurons does not alter synaptic vesicles in nerve terminals. Quantitative comparison showing that the number of synaptic vesicles is similar in nerve terminals from control neurons and from neurons treated with either of three different shRNAs for VPS35 (reproduced from Vazquez-Sanchez et al., 2018 with permission: http://creativecommons.org/licenses/by/4.0/).

\section{RETROMER IN THE ADULT AND DEVELOPING NERVOUS SYSTEM}

Retromer components are ubiquitously expressed in the nervous system. The expression level varies between different brain regions and cell types (Wen et al., 2011; Wang et al., 2012; Lucin et al., 2013; Liu et al., 2014; Tsika et al., 2014; Appel et al., 2018). They occur in association with endosomes in the neuronal cell body and in processes (Wen et al., 2011; Bhalla et al., 2012; Mikhaylova et al., 2016). At synapses they are found in both the pre- and postsynaptic compartments (Jakobsson et al., 2011; Bhalla et al., 2012; Choy et al., 2014; Inoshita et al., 2017; Vazquez-Sanchez et al., 2018). Notably, in postsynaptic dendrites, retromer-bearing endosomes are associated with Golgi satellites, which mediate anterograde protein traffic from the ER as well as retrograde traffic from the plasma membrane (Mikhaylova et al., 2016).

In the developing brain the expression level of VPS35 peaks at stages P10-P15 and then declines to a low level that remains stable during adulthood (Wang et al., 2012). Accordingly, retromer plays a critical role in nervous system development. Full knockout of VPS35 causes death prior to neurogenesis, while heterozygous knockout, or in utero knockdown at a late embryonic stage, leads to impaired development of axons and dendrites (Wen et al., 2011; Wang et al., 2012; Tian et al., 2015). At least some of the developmental defects are due to loss of retromer present in microglia (Appel et al., 2018). The precise mechanisms by which retromer influence mammalian CNS development are not yet clear, but distinct retromer-dependent developmental signaling pathways have been identified in Drosophila and C. elegans (Wang and Bellen, 2015).

\section{RETROMER AND PRESYNAPTIC PROTEIN TRAFFICKING}

Protein trafficking in presynaptic terminals is dominated by synaptic vesicle proteins, which are inserted into the 
plasma membrane upon exocytic neurotransmitter release and subsequently retrieved by endocytosis (Shupliakov and Brodin, 2010). Whether or not the retrieval is followed by an endosomal sorting step prior to vesicle re-use is a classical problem that has been discussed for decades (Heuser and Reese, 1973; Jähne et al., 2015; Milosevic, 2018). Endosome-like structures do indeed form in stimulated nerve terminals, but in many cases they represent plasma membrane invaginations (Figure 1B) or endosome-like objects resulting from ultrafast endocytosis rather than bona fide endosomes (Gad et al., 1998; Kononenko et al., 2014; Watanabe et al., 2014; Jähne et al., 2015; Gan and Watanabe, 2018). Compelling evidence for endosomal sorting of synaptic vesicle components is currently limited to the larval Drosophila neuromuscular junction. In this model, synaptic vesicle recycling has been shown to involve presynaptic endosomes, identified by their characteristic lipid and protein composition (Wucherpfennig et al., 2003; Uytterhoeven et al., 2011). Moreover, enhancement of the endosomal recycling route increases the sorting away of ubiquitin-tagged synaptic vesicle proteins from presynaptic terminals (Uytterhoeven et al., 2011).

Two studies of retromer function at presynaptic terminals have recently shed new light on the problem of endosome involvement in synaptic vesicle recycling. In the first study, Inoshita and co-authors examined the effect of deleting VPS35 in Drosophila (Inoshita et al., 2017). They examined the effect in mutant larvae that survived to a prepupal stage due to the supply of some maternal VPS35. Ultrastructural analysis of VPS35-deficient motor terminals revealed that the number of synaptic vesicles was reduced while their size was increased and was more variable (Figure 1C). The morphological changes correlated with an enhanced fatigue of synaptic transmission. These data thus corroborate the involvement of endosomes in synaptic vesicle recycling in Drosophila larvae, and provide the first evidence for a functional role of retromer in the synaptic vesicle cycle. In the second study, the effects of knocking down VPS35 in mouse hippocampal neurons was investigated (Vazquez-Sanchez et al., 2018). To circumvent developmental defects the knockdown was performed when synaptogenesis was essentially completed. Notably, ultrastructural analysis did not reveal any difference in the number of synaptic vesicles between control and VPS35-depleted nerve terminals (Figure 1D). Nor did the knockdown affect synaptic exo- or endocytosis, which were monitored with a $\mathrm{pH}$-sensitive reporter (Vazquez-Sanchez et al., 2018).

In view of these apparently contradictory data it is unavoidable to speculate that the developmental stage is a critical factor. Studies in toad and mouse motorneurons, for example, suggest that synapse maturation can involve a switch from endosome-dependent to endosome-independent vesicle recycling modes (Zakharenko et al., 1999; Shetty et al., 2013). If this is true, the retromer system in mature nerve terminals can be assumed to serve other functions than to sort synaptic vesicle proteins. In this context it is interesting to note that Vazquez-Sanchez et al. (2018) detected VPS35 in some but not all hippocampal nerve terminals. Investigation of the role of retromer in different synapse types, and at different developmental stages will be of great interest for further studies.

Another presynaptic endosomal system of considerable physiological and pathological importance consists of signaling endosomes. Such endosomes take part in sorting and retrograde axonal transport of endogenous proteins like BDNF and its receptors TrkB and p75NTR, and exogenous agents like Tetanus toxin (Deinhardt et al., 2006; Shupliakov and Fernandez-Chacon, 2008; Surana et al., 2018). Notably, they are also enriched in proteins linked with neurodegenerative disorders (Debaisieux et al., 2016). Whether or not signaling endosomes utilize retromer currently remains an open question.

\section{RETROMER AND NEUROTRANSMITTER TRANSPORTERS}

The plasma membrane dopamine transporter (DAT) acts to terminate DA transmission primarily by mediating reuptake into dopaminergic presynaptic terminals. DA reuptake is affected by psychostimulants such as cocaine and amphetamine, and altered reuptake has been linked with different neuropsychiatric conditions (Sawa and Snyder, 2002; Kristensen et al., 2011; Sharma and Couture, 2014). The level of DAT at the presynaptic plasma membrane is finely tuned by endocytosis followed by either degradation or recycling back to the plasma membrane. Recently, Wu et al. (2017) showed that retromer plays a key role in DAT handling. Newly endocytosed DAT was observed to enter retromer-positive endosomes, and knockdown of VPS35 decreased DAT recycling leading to reduced plasma membrane levels. Moreover, the increase of plasma membrane DAT levels induced by cocaine (Little et al., 2002) could be linked with enhanced recycling of DAT out of retromer-positive endosomes (Wu et al., 2017). Whether other neurotransmitter transporters are sorted by retromer remains to be investigated.

\section{RETROMER AND IONOTROPIC NEUROTRANSMITTER RECEPTORS}

The postsynaptic compartment is a hotspot for trafficking of neurotransmitter receptors. With regard to ionotropic receptors detailed studies have primarily concerned glutamate receptors, which mediate most fast synaptic communication in the brain. In particular, the AMPA receptor subtype, made up of GluA1-4 subunits, has been thoroughly examined (Pick and Ziff, 2018). AMPA receptors are supplied to the postsynaptic membrane by two principal routes. One comprises constitutive delivery whereby receptors are inserted at extrasynaptic sites and then move into synapses by diffusion. The other is a triggered mechanism, which is used to insert receptors into the postsynaptic plasma membrane upon induction of long-term potentiation (LTP), and to remove them upon induction of long-term depression (LTD; Lüscher et al., 2000). In immature hippocampal neurons the constitutive delivery pathway depends on retromer. Thus, heterozygous knockout of VPS35 leads to a reduction of excitatory synaptic transmission along with a reduced amount of AMPA receptors in synaptosomes (Tian 

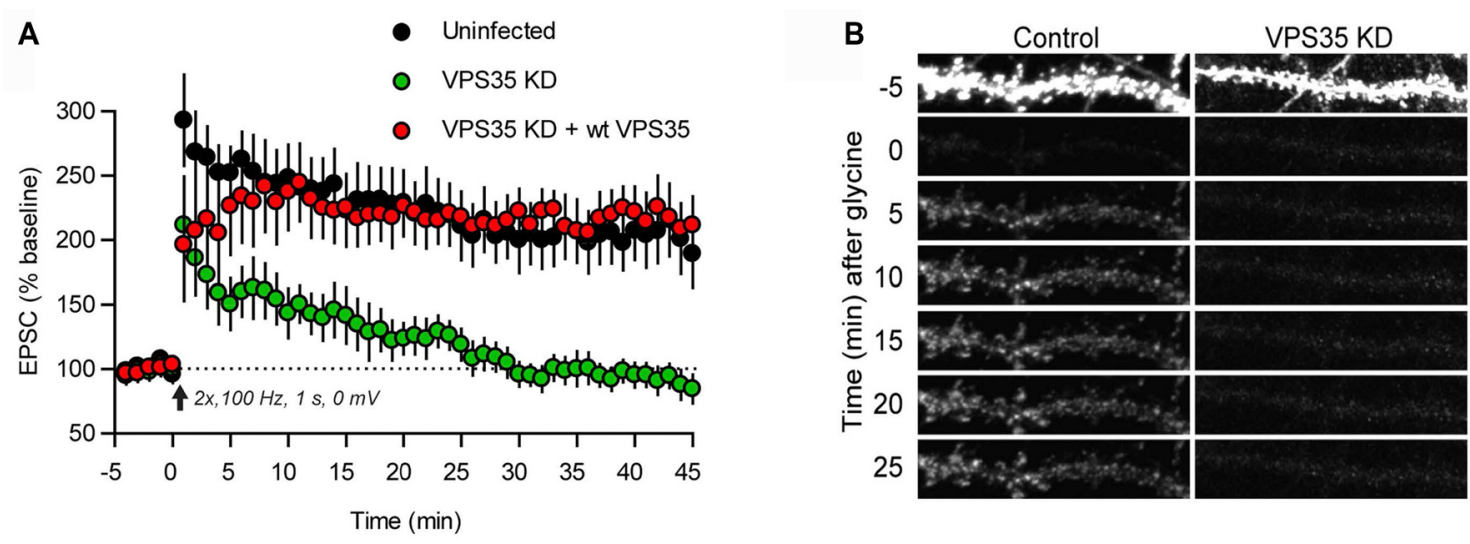

FIGURE 2 | Retromer supports AMPA receptor trafficking during long-term potentiation (LTP). (A) Plot of the excitatory postsynaptic current (EPSC) amplitude during induction of LTP in control hippocampal slices (black dots) and hippocampal slices infected with lentivirus expressing an shRNA for VPS35 (green dots), and a further control, in which VPS35 expression had been restored (red dots). (B) Induction of chemical LTP causes incorporation of GluA1 receptors (tagged with a pH-sensitive reporter) in the dendritic plasma membrane of a hippocampal control neuron (left). The dendrite was photobleached prior to LTP induction to reveal the successive accumulation of GluA1 receptors. Knockdown of VPS35 (right) effectively inhibited receptor incorporation (reproduced from Temkin et al., 2017 with permission).

et al., 2015; see also Choy et al., 2014). Knockout of SNX6 or SNX27 similarly reduces AMPA receptor trafficking to the postsynapse (Hussain et al., 2014; Loo et al., 2014; Niu et al., 2017). In mature neurons, however, the role of retromer is restricted to the triggered pathway. Knockdown of VPS35 in the CA1 region of hippocampus at P21 does not affect basal synaptic transmission but causes an efficient blockade of LTP (Figure 2A; Temkin et al., 2017). Imaging of tagged AMPA receptor subunits suggested that retromer mediates the exit of AMPA receptors into "LTP-ready" vesicles that fuse with the dendritic plasma membrane (Figure 2B). The induction of LTD was not affected by retromer depletion (Temkin et al., 2017).

It should be noted that inhibitory synaptic transmission via GABA receptors is unaffected by retromer depletion even in immature neurons (Choy et al., 2014), thus suggesting a differential regulation of excitatory and inhibitory receptors.

\section{RETROMER AND G-PROTEIN COUPLED NEUROTRANSMITTER RECEPTORS}

G-protein coupled receptors (GPCRs) comprise a large and heterogenous group that induce a wide variety of intracellular signals, mainly via G-proteins or $\beta$-arrestin. Recent studies suggest that retromer plays a key role in the transduction of GPCR signals. This is partly due to the fact that GPCRs, unlike ionotropic receptors, act not only at the plasma membrane but continue to signal at intracellular sites, including endosomes and the TGN (Eichel and von Zastrow, 2018). The localization of GPCRs to these internal compartments typically gives rise to a slower and more prolonged response as compared to plasma membrane localization. Moreover, the type of signal can also be affected. For example, retromer-dependent localization of TSH receptors to the TGN activates a transcriptional response (CREB phosphorylation) that is not seen when the receptor remains at the plasma membrane (Godbole et al., 2017). Similarly, localization of $\beta 2$ adrenergic receptors and dopamine
D1 receptors to endosomes promotes transcriptional responses (Tsvetanova and von Zastrow, 2014; Varandas et al., 2016). In so far most studies of spatial factors and retromer in GPCR signaling have been conducted in cell lines, but future studies in neurons with many of their receptors located in distal dendrites and nerve terminals is likely to give a new level of insight into neuronal GPCR communication. The clinical importance of localized GPCR signaling has recently been underscored by the observation of a spatial signaling difference between opioid drugs and the corresponding native peptide ligands (Stoeber et al., 2018).

Retromer can also regulate the plasma membrane levels of GPCRs (including D1 and $\beta 2$ receptors) by recycling them back from endosomes (Choy et al., 2014; Wang et al., 2016). Imaging studies in striatal medium spiny neurons have elegantly tracked the export of $\beta 2$ receptors from retromer-bearing endosomes that move in the vicinity of postsynaptic densities (Choy et al., 2014). Moreover, GPCR signaling can be regulated by retromer in a more direct way. The CSC component VPS26, which is structurally similar to $\beta$-arrestin, can terminate GPCR signaling by displacing $\beta$-arrestin from the GPCR (Seaman, 2018).

\section{LINKS TO NEURODEGENERATIVE DISORDERS}

Retromer has gained wide interest in recent years due to its involvement in neurodegenerative disorders (Small and Petsko, 2015; Li et al., 2016; McMillan et al., 2017; Williams et al., 2017; Reitz, 2018; Vagnozzi and Praticò, 2018; Zhang et al., 2018). With regard to Alzheimer's disease (AD), the first evidence came from protein profiling studies showing a reduction of VPS26 and VPS35 in brain regions affected by the disease (Small et al., 2005). Genetic studies have subsequently coupled AD with a number of retromer-associated proteins, including SNX1, SNX3, rab7a and SORL1/SORLA (Vardarajan et al., 2012; Lambert et al., 2013; Reitz, 2018). The latter is a retromer receptor that binds to and 
removes the amyloid precursor protein (APP) from endosomes (Eggert et al., 2018). Experimental studies have shown that depletion of VPS35 enhances amyloid $\beta$ peptide $(A \beta)$ production by prolonging the endosomal residence time of APP (Bhalla et al., 2012). Conversely, enhancement of retromer function with a pharmacological chaperone can reduce $A \beta$ formation (Mecozzi et al., 2014), as can overexpression of SNX3 (Xu et al., 2018). The endosomal trafficking of the rate-limiting enzyme in $\mathrm{A} \beta$ production, BACE1, also depends on retromer (Wen et al., 2011; Wang et al., 2012; Toh et al., 2017).

Adding to the link between retromer and APP processing, indirect evidence also suggests an involvement of retromer in Tau pathology, which is another hallmark of AD. The delivery of cathepsin D to lysosomes is retromer-dependent and cathepsin $\mathrm{D}$ deficiency has been shown to aggravate Tau toxicity (Small and Petsko, 2015). Moreover, retromer stabilization can reduce pathology-associated Tau phosphorylation (Young et al., 2018).

In spite of the links with $A \beta$ and Tau pathology, the precise role of retromer in $\mathrm{AD}$ pathogenesis is not fully clear. The question of whether synaptic retromer systems are involved remains open (Figure 3). Synapse loss is a hallmark of $\mathrm{AD}$ that occurs early in disease progression (Masliah et al., 2001; Scheff et al., 2006), and both $A \beta$ and Tau pathologies have been linked with synapses. Thus, $A \beta$ is produced locally at synapses (Dolev et al., 2013; Lundgren et al., 2014, 2015; Das et al., 2016; Schedin-Weiss et al., 2016), and A $\beta$ oligomers

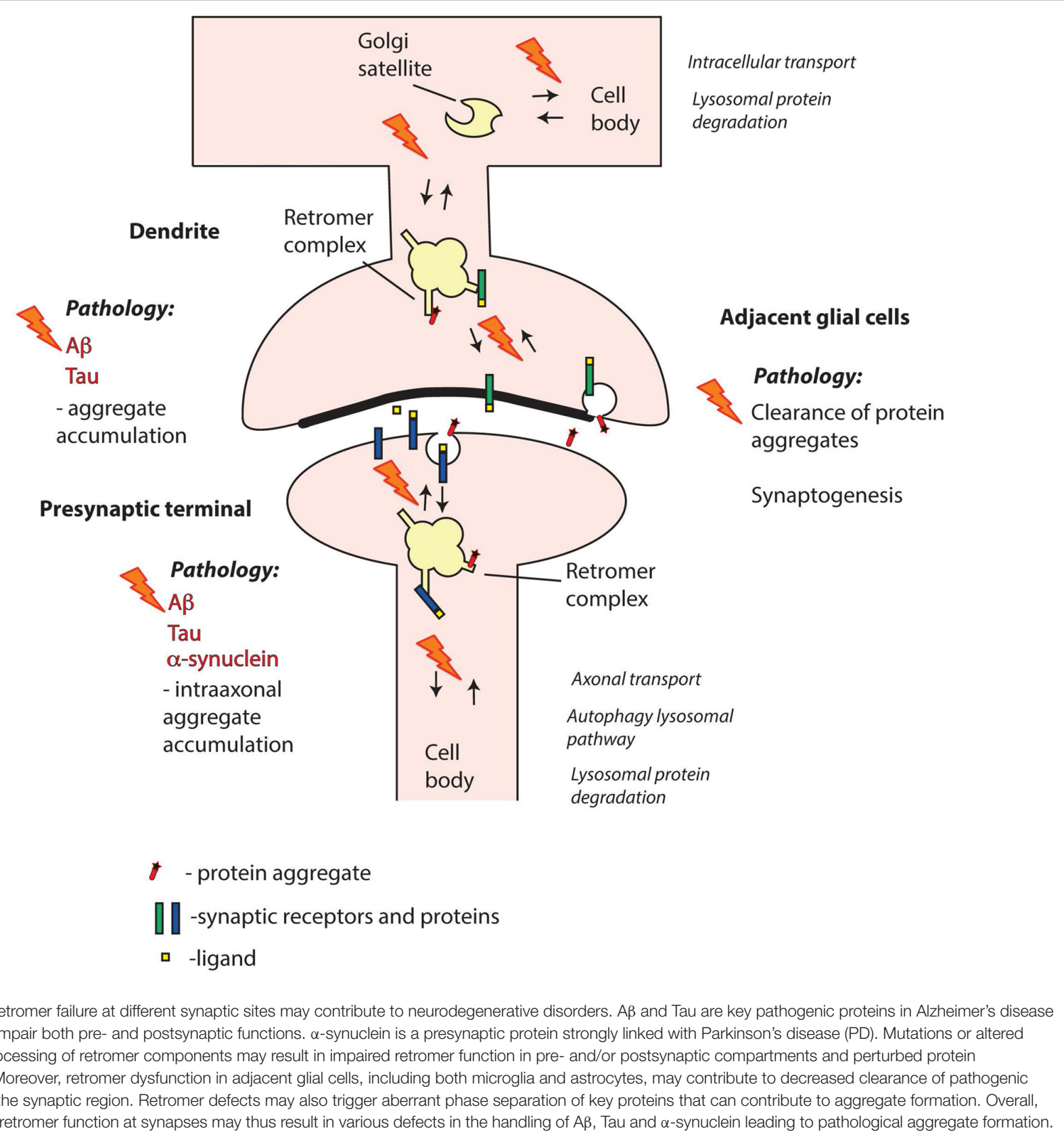


exert toxic effects both pre- and postsynaptically (Ovsepian et al., 2018). Hyperphosphorylated and misfolded Tau oligomers accumulate at pre- and postsynaptic sites at early disease stages (Spires-Jones and Hyman, 2014; Tai et al., 2014), and different Tau forms can disrupt pre- and postsynaptic functions (Hoover et al., 2010; Ittner et al., 2010; Zhou et al., 2017; McInnes et al., 2018). The prion-like spread of Tau pathology between brain regions (Braak and Braak, 1991) appears to occur via synapses, and seed-competent Tau aggregates have been found enriched in synaptosomes (Chang et al., 2018; DeVos et al., 2018). Taken together, these observations point at synaptic retromer systems as possible players in early $\mathrm{AD}$ pathogenesis.

Genetic evidence implicates retromer also in Parkinson's disease (PD). A missense mutation in VPS35, D620N, has been found to cause late-onset $\mathrm{PD}$ in several patient populations world-wide (Williams et al., 2017; Cui et al., 2018). The D620N mutation affects the interaction between VPS35 and the WASH complex, which has multiple effects on endosomal traffic (Seaman and Freeman, 2014; McMillan et al., 2017). The precise link with PD pathogenesis still remains unclear. It has, for instance, been suggested that retromer dysfunction may impair the clearance of $\alpha$-synuclein aggregates, a hallmark of PD, either by impairing the delivery of cathepsin $\mathrm{D}$ (via the retromer receptors CI-MPR or SORLA) to lysosomes, or of autophagyrelated protein 9a to autophagosome precursors (Follett et al., 2014; Zavodszky et al., 2014; Small and Petsko, 2015; Cui et al., 2018). It has also been suggested that connections between retromer and different PD-associated gene products, like LRRK2, Parkin and PLA2G6, are of importance, or that mitochondrial defects play a role (Small and Petsko, 2015; Williams et al., 2017; Lin et al., 2018; Williams et al., 2018).

Similar to the case with AD, some forms of $\mathrm{PD}$ are strongly linked with synapses, and primarily with the presynaptic compartment. Evidence from postmortem and neuroimaging studies in humans along with animal model data suggest that the degeneration of substantia nigra DA neurons may originate in their projections to striatum rather than in the cell bodies (Burke and O'Malley, 2013; Kordower et al., 2013; Laguna et al., 2015; Schirinzi et al., 2016; Pan et al., 2018; Soukup et al., 2018). Among the proteins that have been linked to early PD pathology, $\alpha$-synuclein has been most extensively studied. This protein is normally accumulated in nerve terminals where it is associated with synaptic vesicles (Burré et al., 2017). Its different pathological forms-oligomers, protofibrils and aggregates-can exert toxic effects in nerve terminals (Burré et al., 2017; Bridi and Hirth, 2018). $\alpha$-synuclein pathology may spread in the brain when it occurs in the extracellular space and it has been suggested that synapses are involved in this process via secretion and/or uptake of $\alpha$-synuclein aggregates (Volpicelli-Daley and Brundin, 2018).

The composition and roles of the retromer system in nigrostriatal nerve terminals yet waits to be defined (apart from being implicated in DAT handling as discussed above). Its functional importance is supported by the observation that a mutation in VPS35, D620N, leads to altered DA turnover in striatum (Ishizu et al., 2016; Cataldi et al., 2018).
Other neurodegenerative diseases linked with retromer include Down syndrome, a variant of hereditary spastic paraplegia, and neuronal ceroid lipofuscinoses (Small and Petsko, 2015; Zhang et al., 2018). With regard to Down syndrome, the disease mechanism may be similar to that in $\mathrm{AD}$ as the expression of APP (located at chromosome 21) and A $\beta$ production are enhanced. Moreover, the expression of miR 155 is enhanced causing a reduction of SNX27 expression that can both compromise synaptic glutamate receptor traffic (Wang et al., 2013) and interfere with APP processing (Zhang et al., 2018).

\section{CONCLUSIONS AND FUTURE PERSPECTIVES}

Although the importance of retromer at synapses is beginning to become evident, the field is yet at an early stage and many questions remain to be answered. First and foremost, the scheme of endosomal cargo retrieval vs. degradation (Figure 1A) has been worked out in compact cell bodies and its correlates in distantly located synapses remain largely unexplored. Moreover, insights into neuronal retromer functions are in most cases limited to a handful of neuron types or to extrapolation from cell line studies. A clear priority is thus to expand the study to a broader set of neuron types. This is particularly true for presynaptic retromer systems of which the functions are least well understood. Another priority is to define more precisely the dynamic localization and composition of retromer systems and their accessory proteins in distinct types of synapses. High resolution imaging of these protein complexes at synapses under different conditions will be one of the challenges. Knowledge about putative synapse-specific accessory proteins will permit directed functional studies, and may also facilitate pharmacological development directed at e.g., GPCRs and neurotransmitter transporters.

As yet, direct evidence connecting pre- or postsynaptic retromer systems with pathology are not at hand but, as discussed above, there are many plausible links (Figure 3). Adding to these, another possible connection has recently emerged, which is related to phase separation of proteins (Gomes and Shorter, 2018). Recent evidences indicate that the functional organization of different proteins in the presynaptic (Milovanovic et al., 2018) as well as postsynaptic (Zeng et al., 2016) compartment depends on liquid-liquid phase separation. If phase separation would apply to intracellular pools of for example $\alpha$-synuclein, or even $\mathrm{A} \beta$ and Tau, it may be envisioned that subtle defects in synaptic retromer-dependent protein clearance could initiate a seeding process eventually leading to transition from soluble proteins to insoluble aggregates.

Future experiments focused on the link between synaptic retromer systems and the synapse pathology in $\mathrm{AD}$ and $\mathrm{PD}$ may proceed along different lines. One may relate to improved knowledge about synapse-specific accessory proteins. It would, for example, be of key interest to identify negative regulators that could be used as drug targets. Another line may focus on retromer-stabilizing pharmacological chaperones (Mecozzi et al., 2014). Ways to target such agents to synapses could potentially enhance protective effects and reduce side-effects. 
Moreover, elucidating the molecular basis of the reduction of retromer components in sporadic AD (Small et al., 2005) may lead to new principles that address the basis of the synapse loss.

\section{AUTHOR CONTRIBUTIONS}

Both authors have contributed to ideas and writing of the manuscript.

\section{REFERENCES}

Appel, J. R., Ye, S., Tang, F., Sun, D., Zhang, H., Mei, L., et al. (2018). Increased microglial activity, impaired adult hippocampal neurogenesis and depressive-like behavior in microglial VPS35-depleted mice. J. Neurosci. 38, 5949-5968. doi: 10.1523/jneurosci.3621-17.2018

Bhalla, A., Vetanovetz, C. P., Morel, E., Chamoun, Z., Di Paolo, G., and Small, S. A. (2012). The location and trafficking routes of the neuronal retromer and its role in amyloid precursor protein transport. Neurobiol. Dis. 47, 126-134. doi: $10.1016 /$ j.nbd.2012.03.030

Braak, H., and Braak, E. (1991). Neuropathological stageing of Alzheimer-related changes. Acta Neuropathol. 82, 239-259. doi: 10.1007/bf00308809

Bridi, J. C., and Hirth, F. (2018). Mechanisms of $\alpha$-synuclein induced synaptopathy in Parkinson's disease. Front. Neurosci. 12:80. doi: 10.3389/fnins.2018.00080

Burke, R. E., and O'Malley, K. (2013). Axon degeneration in Parkinson's disease. Exp. Neurol. 246, 72-83. doi: 10.1016/j.expneurol.2012.01.011

Burré, J., Sharma, M., and Südhof, T. C. (2017). Cell biology and pathophysiology of $\alpha$-synuclein. Cold Spring Harb. Perspect. Med. 8:a024091. doi: 10.1101/cshperspect.a024091

Cataldi, S., Follett, J., Fox, J. D., Tatarnikov, I., Kadgien, C., Gustavsson, E. K., et al. (2018). Altered dopamine release and monoamine transporters in Vps35 p.D620N knock-in mice. NPJ Parkinsons Dis. 4:27. doi: 10.1038/s41531018-0063-3

Chang, H.-Y., Sang, T.-K., and Chiang, A.-S. (2018). Untangling the tauopathy for Alzheimer's disease and Parkinsonism. J. Biomed. Sci. 25:54. doi: 10.1186/s12929-018-0457-x

Choy, R. W. Y., Park, M., Temkin, P., Herring, B. E., Marley, A., Nicoll, R. A., et al. (2014). Retromer mediates a discrete route of local membrane delivery to dendrites. Neuron 82, 55-62. doi: 10.1016/j.neuron.2014.02.018

Cui, Y., Yang, Z., and Teasdale, R. D. (2018). The functional roles of retromer in Parkinson's disease. FEBS Lett. 592, 1096-1112. doi: 10.1002/1873-3468.12931

Das, U., Wang, L., Ganguly, A., Saikia, J. M., Wagner, S. L., Koo, E. H., et al. (2016). Visualizing APP and BACE-1 approximation in neurons yields insight into the amyloidogenic pathway. Nat. Neurosci. 19, 55-64. doi: 10.1038/nn.4188

Debaisieux, S., Encheva, V., Chakravarty, P., Snijders, A. P., and Schiavo, G. (2016). Analysis of signaling endosome composition and dynamics using SILAC in embryonic stem cell-derived neurons. Mol. Cell. Proteomics 15, 542-557. doi: $10.1074 / \mathrm{mcp} . \mathrm{m} 115.051649$

Deinhardt, K., Salinas, S., Verastegui, C., Watson, R., Worth, D., Hanrahan, S., et al. (2006). Rab5 and Rab7 control endocytic sorting along the axonal retrograde transport pathway. Neuron 52, 293-305. doi: 10.1016/j.neuron.2006. 08.018

DeVos, S. L., Corjuc, B. T., Oakley, D. H., Nobuhara, C. K., Bannon, R. N., Chase, A., et al. (2018). Synaptic tau seeding precedes tau pathology in human Alzheimer's disease brain. Front. Neurosci. 12:267. doi: 10.3389/fnins.2018. 00267

Dolev, I., Fogel, H., Milshtein, H., Berdichevsky, Y., Lipstein, N., Brose, N., et al. (2013). Spike bursts increase amyloid- $\beta 40 / 42$ ratio by inducing a presenilin-1 conformational change. Nat. Neurosci. 16, 587-595. doi: 10.1038/nn.3376

Eggert, S., Thomas, C., Kins, S., and Hermey, G. (2018). Trafficking in Alzheimer's disease: modulation of APP transport and processing by the transmembrane proteins LRP1, SorLA, SorCS1c, sortilin and calsyntenin. Mol. Neurobiol. 55, 5809-5829. doi: 10.1007/s12035-017-0806-x

Eichel, K., and von Zastrow, M. (2018). Subcellular organization of GPCR signaling. Trends Pharmacol. Sci. 39, 200-208. doi: 10.1016/j.tips.2017. 11.009

\section{FUNDING}

This work was supported by the Swedish Research Council, Hjärnfonden, Parkinsonfonden and the RSF grant 16-15-1023.

\section{ACKNOWLEDGMENTS}

We thank Dr. Shaohua Xu for help with Figure 1.

Follett, J., Norwood, S. J., Hamilton, N. A., Mohan, M., Kovtun, O., Tay, S., et al. (2014). The Vps35 D620N mutation linked to Parkinson's disease disrupts the cargo sorting function of retromer. Traffic 15, 230-244. doi: 10.1111/tra.12136

Gad, H., Löw, P., Zotova, E., Brodin, L., and Shupliakov, O. (1998). Dissociation between $\mathrm{Ca}^{2+}$-triggered synaptic vesicle exocytosis and clathrin-mediated endocytosis at a central synapse. Neuron 21, 607-616. doi: 10.1016/s08966273(00)80570-x

Gan, Q., and Watanabe, S. (2018). Synaptic vesicle endocytosis in different model systems. Front. Cell. Neurosci. 12:171. doi: 10.3389/fncel.2018.00171

Godbole, A., Lyga, S., Lohse, M. J., and Calebiro, D. (2017). Internalized TSH receptors en route to the TGN induce local Gs-protein signaling and gene transcription. Nat. Commun. 8:443. doi: 10.1038/s41467-017-00357-2

Gomes, E., and Shorter, J. (2018). The molecular language of membraneless organelles. J. Biol. Chem. doi: 10.1074/jbc.tm118.001192 [Epub ahead of print].

Heuser, J. E., and Reese, T. S. (1973). Evidence for recycling of synaptic vesicle membrane during transmitter release at the frog neuromuscular junction. J. Cell Biol. 57, 315-344. doi: 10.1083/jcb.57.2.315

Hoover, B. R., Reed, M. N., Su, J., Penrod, R. D., Kotilinek, L. A., Grant, M. K., et al. (2010). Tau mislocalization to dendritic spines mediates synaptic dysfunction independently of neurodegeneration. Neuron 68, 1067-1081. doi: 10.1016/j. neuron.2010.11.030

Hussain, N. K., Diering, G. H., Sole, J., Anggono, V., and Huganir, R. L. (2014), Sorting nexin 27 regulates basal and activity-dependent trafficking of AMPARs. Proc. Natl. Acad. Sci. U S A 111, 11840-11845. doi: 10.1073/pnas.1412415111

Inoshita, T., Arano, T., Hosaka, Y., Meng, H., Umezaki, Y., Kosugi, S., et al. (2017). Vps35 in cooperation with LRRK2 regulates synaptic vesicle endocytosis through the endosomal pathway in Drosophila. Hum. Mol. Genet. 26, 2933-2948. doi: 10.1093/hmg/ddx179

Ishizu, N., Yui, D., Hebisawa, A., Aizawa, H., Cui, W., Fujita, Y., et al. (2016). Impaired striatal dopamine release in homozygous Vps35 D620N knock-in mice. Hum. Mol. Genet. 25, 4507-4517. doi: 10.1093/hmg/ddw279

Ittner, L. M., Ke, Y. D., Delerue, F., Bi, M., Gladbach, A., van Eersel, J., et al. (2010). Dendritic function of tau mediates amyloid- $\beta$ toxicity in Alzheimer's disease mouse models. Cell 142, 387-397. doi: 10.1016/j.cell.2010.06.036

Jähne, S., Rizzoli, S. O., and Helm, M. S. (2015). The structure and function of presynaptic endosomes. Exp. Cell Res. 335, 172-179. doi: 10.1016/j.yexcr.2015. 04.017

Jakobsson, J., Ackermann, F., Andersson, F., Larhammar, D., Low, P., and Brodin, L. (2011). Regulation of synaptic vesicle budding and dynamin function by an EHD ATPase. J. Neurosci. 31, 13972-13980. doi: 10.1523/jneurosci.128911.2011

Kononenko, N. L., Puchkov, D., Classen, G. A., Walter, A. M., Pechstein, A., Sawade, L., et al. (2014). Clathrin/AP-2 mediate synaptic vesicle reformation from endosome-like vacuoles but are not essential for membrane retrieval at central synapses. Neuron 82, 981-988. doi: 10.1016/j.neuron.2014.05.007

Kordower, J. H., Olanow, C. W., Dodiya, H. B., Chu, Y., Beach, T. G., Adler, C. H., et al. (2013). Disease duration and the integrity of the nigrostriatal system in Parkinson's disease. Brain 136, 2419-2431. doi: 10.1093/brain/awt192

Kovtun, O., Leneva, N., Bykov, Y. S., Ariotti, N., Teasdale, R. D., Schaffer, M., et al. (2018). Structure of the membrane-assembled retromer coat determined by cryo-electron tomography. Nature 561, 561-564. doi: 10.1038/s41586-0180526-z

Kristensen, A. S., Andersen, J., Jorgensen, T. N., Sorensen, L., Eriksen, J., Loland, C. J., et al. (2011). SLC6 neurotransmitter transporters: structure, function, and regulation. Pharmacol. Rev. 63, 585-640. doi: 10.1124/pr.108. 000869 
Laguna, A., Schintu, N., Nobre, A., Alvarsson, A., Volakakis, N., Jacobsen, J. K., et al. (2015). Dopaminergic control of autophagic-lysosomal function implicates Lmxlb in Parkinson's disease. Nat. Neurosci. 18, 826-835. doi: 10.1038/nn.4004

Lambert, J.-C., Ibrahim-Verbaas, C. A., Harold, D., Naj, A. C., Sims, R., Bellenguez, C., et al. (2013). Meta-analysis of 74,046 individuals identifies 11 new susceptibility loci for Alzheimer's disease. Nat. Genet. 45, 1452-1458. doi: 10.1038/ng.2802

Li, C., Shah, S. Z., Zhao, D., and Yang, L. (2016). Role of the retromer complex in neurodegenerative diseases. Front. Aging Neurosci. 8:42. doi: 10.3389/fnagi. 2016.00042

Lin, G., Lee, P. T., Chen, K., Mao, D., Tan, K. L., Zuo, Z., et al. (2018). Phospholipase PLA2G6, a Parkinsonism-associated gene, affects Vps26 and Vps35, retromer function and ceramide levels, similar to $\alpha$-synuclein gain. Cell Metab. 28, 605.e6-618.e6. doi: 10.1016/j.cmet.2018.05.019

Little, K. Y., Elmer, L. W., Zhong, H., Scheys, J. O., and Zhang, L. (2002). Cocaine induction of dopamine transporter trafficking to the plasma membrane. Mol. Pharmacol. 61, 436-445. doi: 10.1124/mol.61.2.436

Liu, W., Tang, F. L., Erion, J., Xiao, H., Ye, J., and Xiong, W. C. (2014). Vps35 haploinsufficiency results in degenerative-like deficit in mouse retinal ganglion neurons and impairment of optic nerve injury-induced gliosis. Mol. Brain 7:10. doi: 10.1186/1756-6606-7-10

Loo, L. S., Tang, N., Al-Haddawi, M., Dawe, G. S., and Hong, W. (2014). A role for sorting nexin 27 in AMPA receptor trafficking. Nat. Commun. 5:3176. doi: $10.1038 /$ ncomms4176

Lucin, K. M., O’Brien, C. E., Bieri, G., Czirr, E., Mosher, K. I., Abbey, R. J., et al. (2013). Microglial beclin 1 regulates retromer trafficking and phagocytosis and is impaired in Alzheimer's disease. Neuron 79, 873-886. doi: 10.1016/j.neuron. 2013.06.046

Lundgren, J. L., Ahmed, S., Schedin-Weiss, S., Gouras, G. K., Winblad, B., Tjernberg, L. O., et al. (2015). ADAM10 and BACE1 are localized to synaptic vesicles. J. Neurochem. 135, 606-615. doi: 10.1111/jnc.13287

Lundgren, J. L., Ahmed, S., Winblad, B., Gouras, G. K., Tjernberg, L. O., and Frykman, S. (2014). Activity-independent release of the amyloid $\beta$-peptide from rat brain nerve terminals. Neurosci. Lett. 566, 125-130. doi: 10.1016/j. neulet.2014.02.050

Lüscher, C., Nicoll, R. A., Malenka, R. C., and Muller, D. (2000). Synaptic plasticity and dynamic modulation of the postsynaptic membrane. Nat. Neurosci. 3, 545-550. doi: 10.1038/75714

Masliah, E., Mallory, M., Alford, M., DeTeresa, R., Hansen, L. A., McKeel, D. W. Jr., et al. (2001). Altered expression of synaptic proteins occurs early during progression of Alzheimer's disease. Neurology 56, 127-129. doi: 10.1212/wnl. 56.1.127

McInnes, J., Wierda, K., Snellinx, A., Bounti, L., Wang, Y. C., Stancu, I. C., et al. (2018). Synaptogyrin-3 mediates presynaptic dysfunction induced by tau. Neuron 97, 823.e8-835.e8. doi: 10.1016/j.neuron.2018.01.022

McMillan, K. J., Korswagen, H. C., and Cullen, P. J. (2017). The emerging role of retromer in neuroprotection. Curr. Opin. Cell Biol. 47, 72-82. doi: 10.1016/j. ceb.2017.02.004

McNally, K. E., and Cullen, P. J. (2018). Endosomal retrieval of cargo: retromer is not alone. Trends Cell Biol. 28, 807-822. doi: 10.1016/j.tcb.2018.06.005

Mecozzi, V. J., Berman, D. E., Simoes, S., Vetanovetz, C., Awal, M. R., Patel, V. M., et al. (2014). Pharmacological chaperones stabilize retromer to limit APP processing. Nat. Chem. Biol. 10, 443-449. doi: 10.1038/nchembio.1508

Mikhaylova, M., Bera, S., Kobler, O., Frischknecht, R., and Kreutz, M. R. (2016). A dendritic golgi satellite between ERGIC and retromer. Cell Rep. 14, 189-199. doi: 10.1016/j.celrep.2015.12.024

Milosevic, I. (2018). Revisiting the role of clathrin-mediated endoytosis in synaptic vesicle recycling. Front. Cell. Neurosci. 12:27. doi: 10.3389/fncel.2018. 00027

Milovanovic, D., Wu, Y., Bian, X., and De Camilli, P. (2018). A liquid phase of synapsin and lipid vesicles. Science 361, 604-607. doi: 10.1126/science.aat5671

Mukadam, A. S., and Seaman, M. N. (2015). Retromer-mediated endosomal protein sorting: the role of unstructured domains. FEBS Lett. 589, 2620-2626. doi: 10.1016/j.febslet.2015.05.052

Niu, Y., Dai, Z., Liu, W., Zhang, C., Yang, Y., Guo, Z., et al. (2017). Ablation of SNX6 leads to defects in synaptic function of CA1 pyramidal neurons and spatial memory. Elife 6:e20991. doi: 10.7554/eLife.20991
Ovsepian, S. V., O’Leary, V. B., Zaborszky, L., Ntziachristos, V., and Dolly, O. J. (2018). Synaptic vesicle cycle and amyloid $\beta$ : biting the hand that feeds. Alzheimers Dement. 14, 502-513. doi: 10.1016/j.jalz.2018.01.011

Pan, P. Y., Zhu, Y., Shen, Y., and Yue, Z. (2018). Crosstalk between presynaptic trafficking and autophagy in Parkinson's disease. Neurobiol. Dis. doi: 10.1016/j. nbd.2018.04.020 [Epub ahead of print].

Pick, J. E., and Ziff, E. B. (2018). Regulation of AMPA receptor trafficking and exit from the endoplasmic reticulum. Mol. Cell. Neurosci. 91, 3-9. doi: 10.1016/j. mcn.2018.03.004

Reitz, C. (2018). Retromer dysfunction and neurodegenerative disease. Curr. Genomics 19, 279-288. doi: 10.2174/1389202919666171024122809

Sawa, A., and Snyder, S. H. (2002). Schizophrenia: diverse approaches to a complex disease. Science 296, 692-695. doi: 10.1126/science.1070532

Schedin-Weiss, S., Caesar, I., Winblad, B., Blom, H., and Tjernberg, L. O. (2016). Super-resolution microscopy reveals $\gamma$-secretase at both sides of the neuronal synapse. Acta Neuropathol. Commun. 4:29. doi: 10.1186/s40478-016 -0296-5

Scheff, S. W., Price, D. A., Schmitt, F. A., and Mufson, E. J. (2006). Hippocampal synaptic loss in early Alzheimer's disease and mild cognitive impairment. Neurobiol. Aging 27, 1372-1384. doi: 10.1016/j.neurobiolaging.2005.09.012

Schirinzi, T., Madeo, G., Martella, G., Maltese, M., Picconi, B., Calabresi, P., et al. (2016). Early synaptic dysfunction in Parkinson's disease: insights from animal models. Mov. Disord. 31, 802-813. doi: 10.1002/mds. 26620

Seaman, M. N. (2012). The retromer complex-endosomal protein recycling and beyond. J. Cell Sci. 125, 4693-4702. doi: 10.1242/jcs.103440

Seaman, M. N. J. (2017). Retromer and the CIMPR-time for a trial separation? Traffic 19, 150-152. doi: 10.1111/tra.12542

Seaman, M. N. J. (2018). Retromer and its role in regulating signaling at endosomes. Prog. Mol. Subcell. Biol. 57, 137-149. doi: 10.1007/978-3-31996704-2_5

Seaman, M. N. J., and Freeman, C. L. (2014). Analysis of the Retromer complex-WASH complex interaction illuminates new avenues to explore in Parkinson disease. Commun. Integr. Biol. 7:e29483. doi: 10.4161/cib.29483

Seaman, M. N. J., Mukadam, A. S., and Breusegem, S. Y. (2018). Inhibition of TBC1D5 activates Rab7a and can enhance the function of the retromer cargoselective complex. J. Cell Sci. 131:jcs217398. doi: 10.1242/jcs.217398

Sharma, A., and Couture, J. (2014). A review of the pathophysiology, etiology and treatment of attention-deficit hyperactivity disorder (ADHD). Ann. Pharmacother. 48, 209-225. doi: 10.1177/1060028013510699

Shetty, A., Sytnyk, V., Leshchyns'ka, I., Puchkov, D., Haucke, V., and Schachner, M. (2013). The neural cell adhesion molecule promotes maturation of the presynaptic endocytotic machinery by switching synaptic vesicle recycling from adaptor protein 3 (AP-3)- to AP-2-dependent mechanisms. J. Neurosci. 33, 16828-16845. doi: 10.1523/JNEUROSCI.2192-13.2013

Shupliakov, O., and Brodin, L. (2010). Recent insights into the building and cycling of synaptic vesicles. Exp. Cell Res. 316, 1344-1350. doi: 10.1016/j.yexcr.2010. 02.035

Shupliakov, O., and Fernandez-Chacon, R. (2008). "Presynaptic endosome," in Encyclopedia of Neuroscience, 4th Edn. ed. L. Squire (Amsterdam: Elsevier), 975-979.

Small, S. A., Kent, K., Pierce, A., Leung, C., Kang, M. S., Okada, H., et al. (2005). Model-guided microarray implicates the retromer complex in Alzheimer's disease. Ann. Neurol. 58, 909-919. doi: 10.1002/ana.20667

Small, S. A., and Petsko, G. A. (2015). Retromer in Alzheimer disease, Parkinson disease and other neurological disorders. Nat. Rev. Neurosci. 16, 126-132. doi: $10.1038 / \mathrm{nrn} 3896$

Soukup, S. F., Vanhauwaert, R., and Verstreken, P. (2018). Parkinson's disease: convergence on synaptic homeostasis. EMBO J. 37:e98960. doi: 10.15252/embj. 201898960

Spires-Jones, T. L., and Hyman, B. T. (2014). The intersection of amyloid $\beta$ and tau at synapses in Alzheimer's disease. Neuron 82, 756-771. doi: 10.1016/j.neuron. 2014.05.004

Stoeber, M., JulliÜ, D., Lobingier, B. T., Laeremans, T., Steyaert, J., Schiller, P. W., et al. (2018). A genetically encoded biosensor reveals location bias of opioid drug action. Neuron 98, 963.e5-976.e5. doi: 10.1016/j.neuron.2018.04.021

Surana, S., Tosolini, A. P., Meyer, I. F. G., Fellows, A. D., Novoselov, S. S., and Schiavo, G. (2018). The travel diaries of tetanus and botulinum neurotoxins. Toxicon 147, 58-67. doi: 10.1016/j.toxicon.2017.10.008 
Tai, H.-C., Wang, B. Y., Serrano-Pozo, A., Frosch, M. P., Spires-Jones, T. L., and Hyman, B. T. (2014). Frequent and symmetric deposition of misfolded tau oligomers within presynaptic and postsynaptic terminals in Alzheimer's disease. Acta Neuropathol. Commun. 2:146. doi: 10.1186/s40478-014-0146-2

Temkin, P., Lauffer, B., Jäger, S., Cimermancic, P., Krogan, N. J., and von Zastrow, M. (2011). SNX27 mediates retromer tubule entry and endosometo-plasma membrane trafficking of signalling receptors. Nat. Cell Biol. 13, 715-721. doi: 10.1038/ncb2252

Temkin, P., Morishita, W., Goswami, D., Arendt, K., Chen, L., and Malenka, R. (2017). The Retromer supports AMPA receptor trafficking during LTP. Neuron 94, 74.e5-82.e5. doi: 10.1016/j.neuron.2017.03.020

Tian, Y., Tang, F. L., Sun, X., Wen, L., Mei, L., Tang, B. S., et al. (2015). VPS35deficiency results in an impaired AMPA receptor trafficking and decreased dendritic spine maturation. Mol. Brain 8:70. doi: 10.1186/s13041-015-0156-4

Toh, W. H., Chia, P. Z. C., Hossain, M. I., and Gleeson, P. A. (2017). GGA1 regulates signal-dependent sorting of BACE1 to recycling endosomes which moderates A $\beta$ production. Mol. Biol. Cell 29, 191-208. doi: 10.1091/mbc. E17-05-0270

Tsika, E., Glauser, L., Moser, R., Fiser, A., Daniel, G., Sheerin, U. M., et al. (2014). Parkinson's disease-linked mutations in VPS35 induce dopaminergic neurodegeneration. Hum. Mol. Genet. 23, 4621-4638. doi: $10.1093 / \mathrm{hmg} / \mathrm{ddu} 178$

Tsvetanova, N. G., and von Zastrow, M. (2014). Spatial encoding of cyclic AMP signaling specificity by GPCR endocytosis. Nat. Chem. Biol. 10, 1061-1065. doi: $10.1038 /$ nchembio. 1665

Uytterhoeven, V., Kuenen, S., Kasprowicz, J., Miskiewicz, K., and Verstreken, P. (2011). Loss of skywalker reveals synaptic endosomes as sorting stations for synaptic vesicle proteins. Cell 145, 117-132. doi: 10.1016/j.cell.2011.02.039

Vagnozzi, A. N., and Praticò, D. (2018). Endosomal sorting and trafficking, the retromer complex and neurodegeneration. Mol. Psychiatry doi: 10.1038/s41380-018-0221-3 [Epub ahead of print].

Varandas, K. C., Irannejad, R., and von Zastrow, M. (2016). Retromer endosome exit domains serve multiple trafficking destinations and regulate local $\mathrm{G}$ protein activation by GPCRs. Curr. Biol. 26, 3129-3142. doi: 10.1016/j.cub.2016. 09.052

Vardarajan, B. N., Bruesegem, S. Y., Harbour, M. E., Inzelberg, R., Friedland, R., St. George-Hyslop, P., et al. (2012). Identification of Alzheimer diseaseassociated variants in genes that regulate retromer function. Neurobiol. Aging 33, 2231.e15-2231.e30. doi: 10.1016/j.neurobiolaging.2012.04.020

Vazquez-Sanchez, S., Bobeldijk, S., Dekker, M. P., van Keimpema, L., and van Weering, J. R. T. (2018). VPS35 depletion does not impair presynaptic structure and function. Sci. Rep. 8:2996. doi: 10.1038/s41598-018-20448-4

Volpicelli-Daley, L., and Brundin, P. (2018). Prion-like propagation of pathology in Parkinson disease. Handb. Clin. Neurol. 153, 321-335. doi: 10.1016/b978-0444-63945-5.00017-9

Wang, S., and Bellen, H. J. (2015). The retromer complex in development and disease. Development 142, 2392-2396. doi: 10.1242/dev.123737

Wang, C., Niu, M., Zhou, Z., Zheng, X., Zhang, L., Tian, Y., et al. (2016). VPS35 regulates cell surface recycling and signaling of dopamine receptor D1. Neurobiol. Aging 46, 22-31. doi: 10.1016/j.neurobiolaging.2016. 05.016

Wang, C.-L., Tang, F.-L., Peng, Y., Shen, C.-Y., Mei, L., and Xiong, W.-C. (2012). VPS35 regulates developing mouse hippocampal neuronal morphogenesis by promoting retrograde trafficking of BACE1. Biol. Open 1, 1248-1257. doi: 10.1242/bio.20122451

Wang, X., Zhao, Y., Zhang, X., Badie, H., Zhou, Y., Mu, Y., et al. (2013). Loss of sorting nexin 27 contributes to excitatory synaptic dysfunction by modulating glutamate receptor recycling in Down's syndrome. Nat. Med. 19, 473-480. doi: $10.1038 / \mathrm{nm} .3117$
Watanabe, S., Trimbuch, T., Camacho-Pérez, M., Rost, B. R., Brokowski, B., Sohl-Kielczynski, B., et al. (2014). Clathrin regenerates synaptic vesicles from endosomes. Nature 515, 228-233. doi: 10.1038/nature13846

Wen, L., Tang, F. L., Hong, Y., Luo, S. W., Wang, C. L., He, W., et al. (2011). VPS35 haploinsufficiency increases Alzheimer's disease neuropathology. J. Cell Biol. 195, 765-779. doi: 10.1083/jcb.201105109

Williams, E. T., Chen, X., and Moore, D. J. (2017). VPS35, the retromer complex and Parkinson's disease. J. Parkinsons Dis. 7, 219-233. doi: 10.3233/JPD-161020

Williams, E. T., Glauser, L., Tsika, E., Jiang, H., Islam, S., and Moore, D. J. (2018). Parkin mediates the ubiquitination of VPS35 and modulates retromer-dependent endosomal sorting. Hum. Mol. Genet. 27, 3189-3205. doi: $10.1093 / \mathrm{hmg} / \mathrm{ddy} 224$

Wu, S., Fagan, R. R., Uttamapinant, C., Lifshitz, L. M., Fogarty, K. E., Ting, A. Y., et al. (2017). The dopamine transporter recycles via a retromer-dependent postendocytic mechanism: tracking studies using a novel fluorophore-coupling approach. J. Neurosci. 37, 9438-9452. doi: 10.1523/JNEUROSCI.3885-16.2017

Wucherpfennig, T., Wilsch-Bräuninger, M., and González-Gaitán, M. (2003). Role of Drosophila Rab5 during endosomal trafficking at the synapse and evoked neurotransmitter release. J. Cell Biol. 161, 609-624. doi: 10.1083/jcb.200211087

Xu, S., Nigam, S. M., and Brodin, L. (2018). Overexpression of SNX3 decreases amyloid- $\beta$ peptide production by reducing internalization of amyloid precursor protein. Neurodegener. Dis. 18, 26-37. doi: 10.1159/000486199

Young, J. E., Fong, L. K., Frankowski, H., Petsko, G. A., Small, S. A., and Goldstein, L. S. B. (2018). Stabilizing the retromer complex in a human stem cell model of Alzheimer's disease reduces TAU phosphorylation independently of amyloid precursor protein. Stem Cell Reports 10, 1046-1058. doi: 10.1016/j. stemcr.2018.01.031

Zakharenko, S., Chang, S., O’Donoghue, M., and Popov, S. V. (1999). Neurotransmitter secretion along growing nerve processes: comparison with synaptic vesicle exocytosis. J. Cell Biol. 144, 507-518. doi: 10.1083/jcb. 144.3.507

Zavodszky, E., Seaman, M. N., Moreau, K., Jimenez-Sanchez, M., Breusegem, S. Y., Harbour, M. E., et al. (2014). Mutation in VPS35 associated with Parkinson's disease impairs WASH complex association and inhibits autophagy. Nat. Commun. 5:3828. doi: 10.1038/ncomms4828

Zeng, M., Shang, Y., Araki, Y., Guo, T., Huganir, R. L., and Zhang, M. (2016). Phase transition in postsynaptic densities underlies formation of synaptic complexes and synaptic plasticity. Cell 166, 1163.e12-1175.e12. doi: 10.1016/j.cell.2016. 07.008

Zhang, H., Huang, T., Hong, Y., Yang, W., Zhang, X., Luo, H., et al. (2018). The retromer complex and sorting nexins in neurodegenerative diseases. Front. Aging Neurosci. 10:79. doi: 10.3389/fnagi.2018.00079

Zhang, J., Naslavsky, N., and Caplan, S. (2012). EHDs meet the retromer: complex regulation of retrograde transport. Cell. Logist. 2, 161-165. doi: 10.4161/cl. 20582

Zhou, L., McInnes, J., Wierda, K., Holt, M., Herrmann, A. G., Jackson, R. J., et al. (2017). Tau association with synaptic vesicles causes presynaptic dysfunction. Nat. Commun. 8:15295. doi: 10.1038/ncomms15295

Conflict of Interest Statement: The authors declare that the research was conducted in the absence of any commercial or financial relationships that could be construed as a potential conflict of interest.

Copyright (C) 2018 Brodin and Shupliakov. This is an open-access article distributed under the terms of the Creative Commons Attribution License (CC BY). The use, distribution or reproduction in other forums is permitted, provided the original author(s) and the copyright owner(s) are credited and that the original publication in this journal is cited, in accordance with accepted academic practice. No use, distribution or reproduction is permitted which does not comply with these terms. 